\title{
Analysis of the phase transformation near the crack tip in Shape Memory Alloys
}

\author{
V. Taillebot ${ }^{1 \mathrm{a}}$, C. Lexcellent ${ }^{1}$, P. Malécot ${ }^{1}$, R. Laydi $^{1}$ \\ Institute Femto-St, 25000 Besancon, France \\ ${ }^{1}$ Applied Mechanics Department, 24 rue de l'Epitaphe, 25000 Besancon, France
}

\begin{abstract}
A new analytical prediction of the phase transformation zone around the crack tip is proposed for shape memory alloys. The present analysis integrates the asymmetry between tension and compression in the yield surface of phase transformation around the crack tip. Shapes and sizes of the phase transformation zone are predicted for loading modes I, II and III. Furthermore, an experimental investigation for mode I loading fracture tests on edgecracked specimens of a nickel-titanium alloy is currently performing with a particular attention devoted on displacement and temperature fields evolution around the crack tip.
\end{abstract}

The use of shape memory alloys (SMAs), for instance smart structures actuators, has a great appeal for industrial applications, mostly in medical device or aeronautics. SMAs own very large recoverable strains (of the order of $8 \%$ for NiTi alloys) as a result of a phase transformation from austenite to martensite. Thus, the investigation of the SMAs fracture is a relevant question in order to improve service life of systems. The linear elastic fracture mechanics (LEFM) theory allows knowing the stress field around the crack tip which depends on the loading mode. Theoretically, the stress field around the crack tip is unbounded. However, as usual elastic materials undergo yielding at the crack tip, SMAs accommodate the applied stress through a material phase change. If a sheet initially in the austenic state is mechanical loaded, applied stress induces the creation of a martensitic phase transformation region in the vicinity of the crack tip. In this case, the crack tip region is governed by the stress field associated to the SMA pseudoelastic behaviour.

\section{Analytical prediction of phase transformation region}

In recent paper, Freed et Banks-Sill [1] rewieved the experimental and theoretical studies on failures. They cited another recent paper, by Robertson and Richie [2], about the fracture toughening behaviour of a NiTi tube which was investigated experimentally. Classical SMAs behaviour models as those from Panoskaltis et al [3] have been used. This model considers the phase transformation surface as de Huber-Von Mises one. This present work aims at determining the shape and the size of the transformation zone in the vicinity of the crack tip with using our model [4,5], taking in account the asymmetry between tension and compression.

\subsection{General formulation of transformation equation}

Let us consider the second order symmetric $a$ and the deviatoric tensor $S_{a}$ defined by

$$
S_{a}=a-\frac{1}{3} \operatorname{tr}(a) 1
$$

where 1 is the identity tensor. Two dots : denotes the scalar product and $\operatorname{tr}($.$) denotes the trace operator, so that$ $a: b=\operatorname{tr}\left(a^{T} b\right)$ for every second orders tensors $a$ and $b$ and $|a|=\sqrt{\operatorname{tr}\left(a^{T} a\right)}$.

\footnotetext{
a e-mail: virginie.tailleboteuniv-fcomte.fr
}

This is an Open Access article distributed under the terms of the Creative Commons Attribution-Noncommercial License (http://creativecommons.org/licenses/by-nc/3.0/), which permits unrestricted use, distribution, and reproduction in any noncommercial medium, provided the original work is properly cited. 
In a classical way, the Huber-Von Mises equivalent stress $\bar{\sigma}$ is defined as $\bar{\sigma} \equiv \kappa\left|S_{\sigma}\right|$, with $\kappa \equiv \sqrt{\frac{3}{2}}$ the normalisation parameter. The lode invariant (third invariant) $y_{a}$ chosen for instance by Raniecki and Lexcellent is defined by

$$
y_{a} \equiv \frac{6 \kappa}{\left|S_{a}\right|^{3}} \operatorname{det}\left(S_{a}\right)
$$

Whatever the chosen mode $I=1,2$ or 3 , the stress tensor $\sigma_{M(r, \theta)}$ (obtained for LEFM theory) can be decomposed as

$$
\sigma_{M(r, \theta)}=\chi_{I}(r) q_{I}(\theta)
$$

where $(r, \theta)$ are the polar coordinates from the crack tip point A origin and origin and

$$
\chi_{I}(r)=\frac{K_{I}}{\sqrt{2 \pi r}}>0
$$

depending of the loading mode $K_{I}=K_{1}, K_{2}$ or $K_{3}$.

Thus,

$$
S_{\sigma}=\chi_{I}(r) S_{q}(\theta)
$$

and the lode invariant $y_{\sigma}$ is independent from the variable $r$

$$
y_{\sigma}(\theta) \equiv \frac{6 \kappa}{\left|S_{q}(\theta)\right|^{3}} \operatorname{det}\left(S_{q}(\theta)\right) .
$$

The Huber-Von Mises equivalent stress $\bar{\sigma}$ can be written as

$$
\bar{\sigma}=\kappa \chi(r)\left|S_{q}(\theta)\right|=\kappa \frac{K_{I}}{\sqrt{2 \pi r}}\left|S_{q}(r)\right|
$$

One obtains the radius $r$ as function of $\theta$

$$
r(\theta)=\frac{1}{2 \pi}\left(\frac{K_{I}}{\bar{\sigma}}\right)^{2} \kappa^{2}\left|S_{q}(\theta)\right|^{2} .
$$

The general form of the phase transformation equation is taken as:

$$
F(\underline{\sigma}, T)=\bar{\sigma} f\left(y_{\sigma}\right)-\sigma_{c}(T)=0 .
$$

where $f>0$ is a smooth function of $y_{\sigma}$ (which the second derivative $f^{\prime \prime}$ is continuous) which is defined in the interval $I_{y_{\omega}}=[-1,1]$. Actually, $f$ is some "correction" function which allows taking into account the asymmetry between tension and compression. $f$ is also called the shape function.

Moreover, is given by

$$
\sigma_{c}(T)=\left\{\begin{array}{llc}
b\left(T-M_{S}^{\circ}\right) & A \rightarrow M & \text { transformation onset }(z=0) \\
b\left(T-M_{F}^{\circ}\right) & A \rightarrow M & \text { transformation end }(\mathrm{z}=1) \\
b\left(T-A_{S}{ }^{\circ}\right) & M \rightarrow A & \text { transformation onset }(\mathrm{z}=1) \\
b\left(T-A_{F}{ }^{\circ}\right) & M \rightarrow A & \text { transformation end }(z=0)
\end{array}\right.
$$

where $M_{S}{ }^{\circ}, M_{F}{ }^{\circ}, A_{S}{ }^{\circ}$ et $A_{F}{ }^{\circ}$ are the phase transformation temperatures measured in a stress-free state. 
The Huber-Von Mises stress can be written as $\bar{\sigma}=\frac{\sigma}{f\left(y_{\sigma}\right)}$ in a previous equation and the radius $r(\theta)$ can be obtained as

$$
r(\theta)=\underbrace{\frac{1}{4 \pi}\left(\frac{K_{I}}{\sigma_{c}}\right)^{2}}_{L_{A m f}}(\underbrace{2 \kappa^{2}\left|S_{q}(\theta)\right|^{2}}_{R(\theta)}) f^{2}\left(y_{\sigma}\right) .
$$

Finally, the adimensionnal $r_{L}$ is defined as

$$
r_{L} \equiv \frac{r}{L_{A m f}}=R(\theta) f^{2}\left(y_{\sigma}\right)
$$

where

$$
\begin{aligned}
& R(\theta)=3\left|S_{q}(\theta)\right|^{2} \\
& L_{\text {Amf }}=\frac{1}{4 \pi}\left(\frac{K_{I}}{\sigma_{c}}\right)^{2} .
\end{aligned}
$$

At last, we introduce the parameter $\beta$ in order to make the distinction between plane stress (CP) and plane strain (DP) conditions

$$
\beta=\left\{\begin{array}{cc}
1 & \mathrm{CP} \\
1-2 v & \mathrm{DP}
\end{array} .\right.
$$

The choice of the shape function is important. The yield function $F(\sigma)$ must be convex in the stress space whatever $\sigma$. Starting from proportional loadings on CuAlBe by Bouvet et al. [6] (bicompression on cubes and traction-compression, internal pressure on tubes), a first expression was chosen in order to fit the experimental results

$$
f\left(y_{\sigma}\right)=\cos \left(\frac{1}{3} \arccos \left(1-a\left(1-y_{\sigma}\right)\right)\right)
$$

Laydi and Lexecellent [7] have shown that $F(\sigma)$ is convex if and only if $0 \leq a \leq 1$. A simplest choice can be also be done by:

with the convexity condition that

$$
f\left(y_{\sigma}\right)=1+b y_{\sigma}
$$

$$
0 \leq b \leq \frac{1}{8}
$$

\subsection{Results}

As in many models, this model employs only one internal variable, the martensite fraction $z$ such $0 \leq z \leq 1$. The three elementary fracture loading modes were investigated: the opening mode $\mathrm{I}$, the shear plane one II and the shear antiplane or tearing mode III. For each one, the plane stress conditions and the plane strain conditions are examined with $v=1 / 3$. To simplify, we assume that the material around the crack tip is fully transformed $(z=1)$ and show the transformation equivalent surface closest to the crack tip. So, $\sigma_{c}$ is taken as:

$$
\sigma_{c}=b\left(T-M_{F}^{\circ}\right) \text {. }
$$

Analytically, the deformed domain can be divided in four regions: an untransformed region $(z=0)$ in the far field, then more and more close to the crack tip, a transformation zone $(0<z<1)$ where austenite has been partially transformed, a fully transformed zone $(z=1)$ and a plastic martensitic zone at the crack tip. At first, the plastic zone of martensite is neglected. Here the presented analytical predictions focus on the transformation end surface border $(z=1)$. 


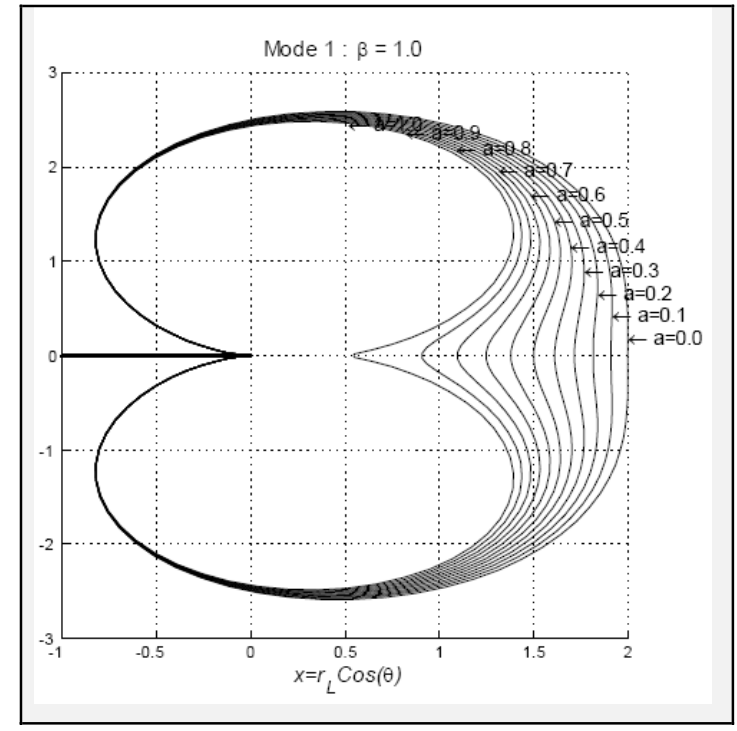

a)

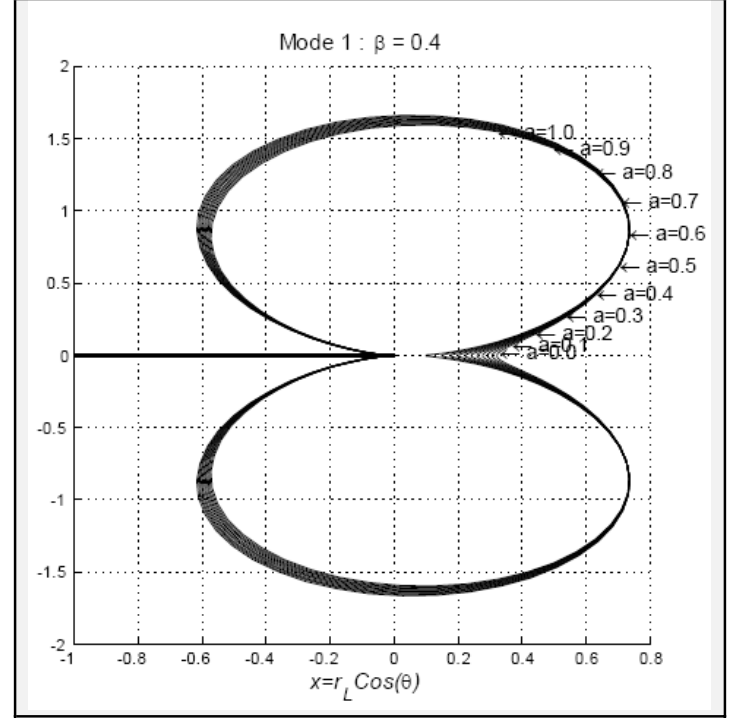

b)

Fig. 1. Mode I, phase transformation surface and parameter $a$ influence in plane stress a) and plane strain conditions b).

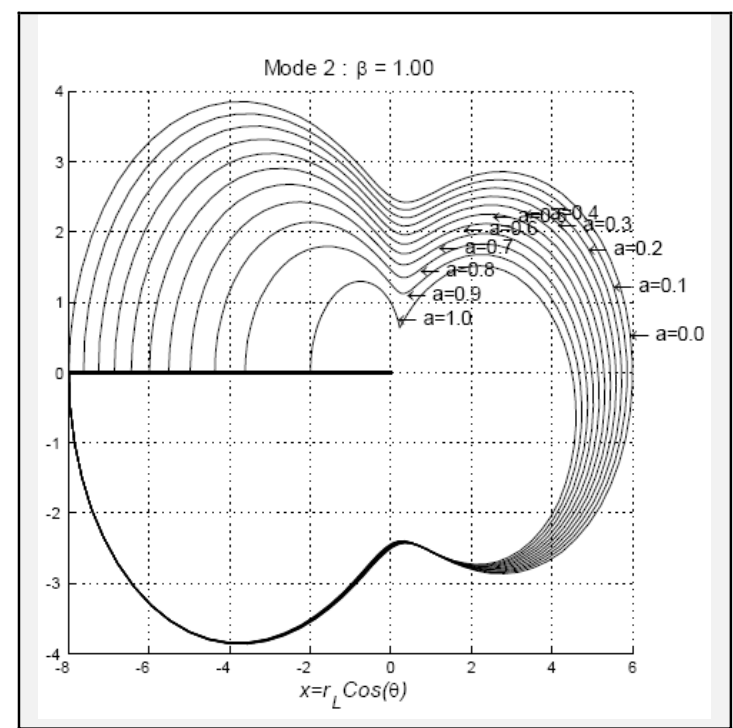

a)

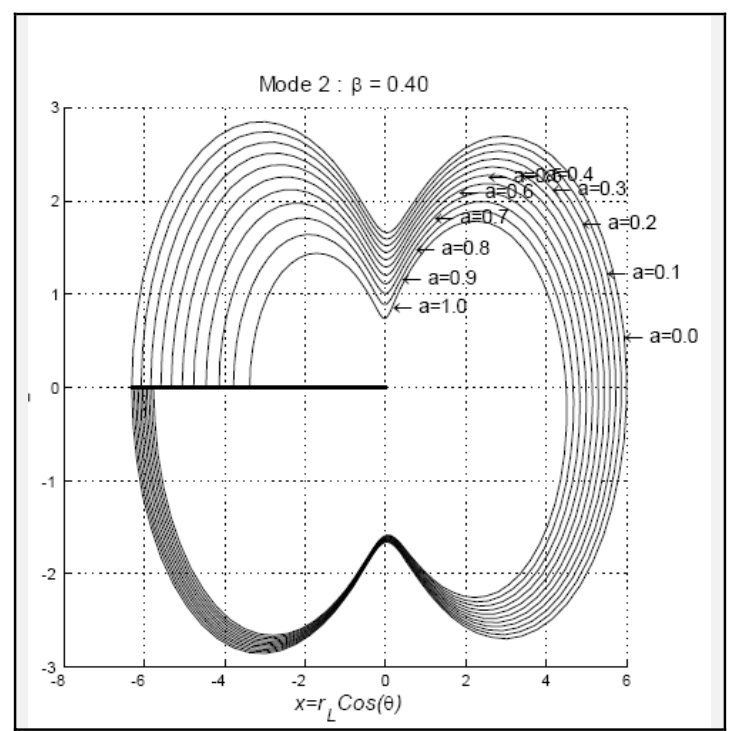

b)

Fig. 2. Mode II, phase transformation surface and parameter $a$ influence in plane stress a) and plane strain conditions b).

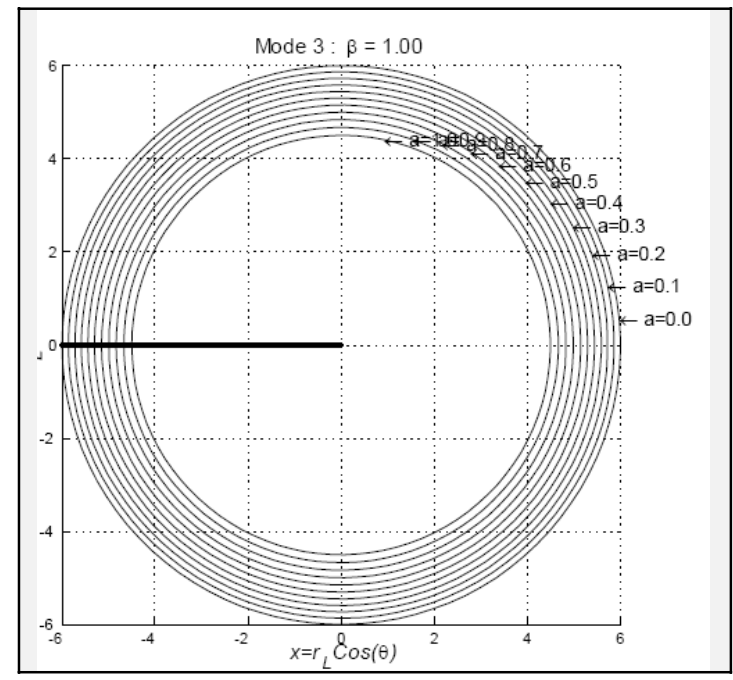

Fig. 3. Mode III, phase transformation surface and parameter $a$ influence in plane stress 
Obviously, for a given mode, the surface shapes have similarities with elasto-plasticity results from LEFM. Moreover, the transformation surface sizes are governed by the parameter $a$. Particularly for mode I, this parameter value, ie the asymmetry between tension and compression, is more decisive in plane stress than in plane strain conditions [8].

\section{Towards an experimental correlation}

Two experimental aspects are developed in this section. An experimental investigation is currently implemented in order to observe strain localization during tip propagation (saturation and transformation regions) then, to correlate analytical predictions and experiments. It consists in measuring both temperature field with an infrared camera and displacement field with images correlation during mode I loading fracture tests.

\subsection{First observations of the temperature field}

Two first fracture tests have been done on rectangular sample $(70 \mathrm{~mm}$ x $29 \mathrm{~mm}$ x $1.25 \mathrm{~mm})$ in NiTi with ${A_{S}}^{\circ}=10^{\circ} \mathrm{C}$ and ${A_{S}}^{\circ}=40^{\circ} \mathrm{C}$. A $10 \mathrm{~mm}$ long sharp edge crack was machined perpendicular to the loading direction by electrical discharge machining. The specimens are tested in uniaxial tension at room temperature, under displacement control using a computer-controlled servo-hydraulic machine (Instron 8501) with a $100 \mathrm{kN}$ load cell and a $150 \mathrm{~mm}$ displacement amplitude. During fracture test, they are deformed at a 0.001 mm.s-1 strain rate and the acquisition frequency is $20 \mathrm{~Hz}$. One of the sample faces is coated with mat black powder to observe thermal flow. The infrared camera is a Jade MWIR one (equipped with a InSb sensor with a $20 \mathrm{mK}$ precision) and placed in front of the black face. For fracture tests, a sampling frequency of $150 \mathrm{~Hz}$ and a subsampling of $1 / 20$ are used. The acquisition and exploitation software ALTAIR permits to parameter data acquisition, and the real-time visualization and images post-processing. Finally, these tests are conducted in very close plane stress conditions. Figure 4 shows temperature field before sample fracture and figure 5, temperature at crack tip during test. Average temperature of the specimen remains quite stable throughout the test. However, local temperature variations are important particularly around the crack tip.

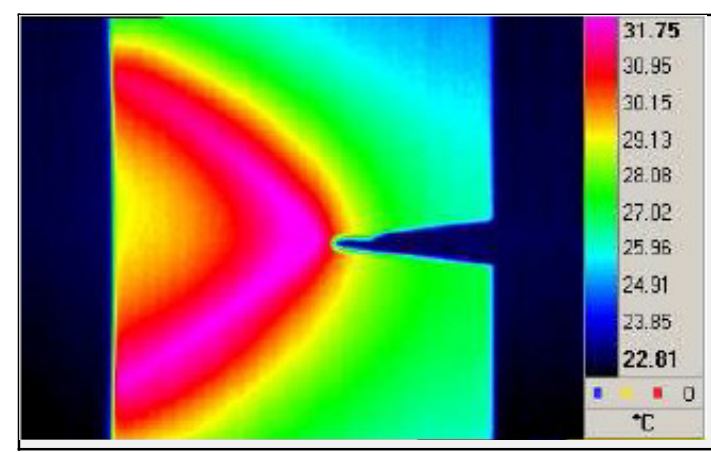

a)

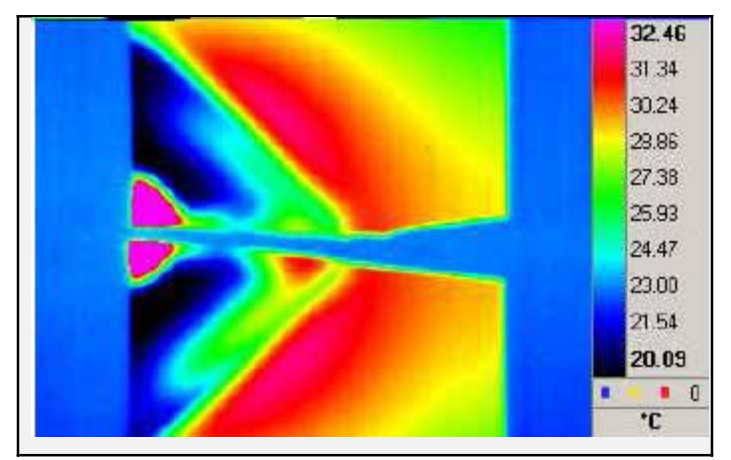

c)

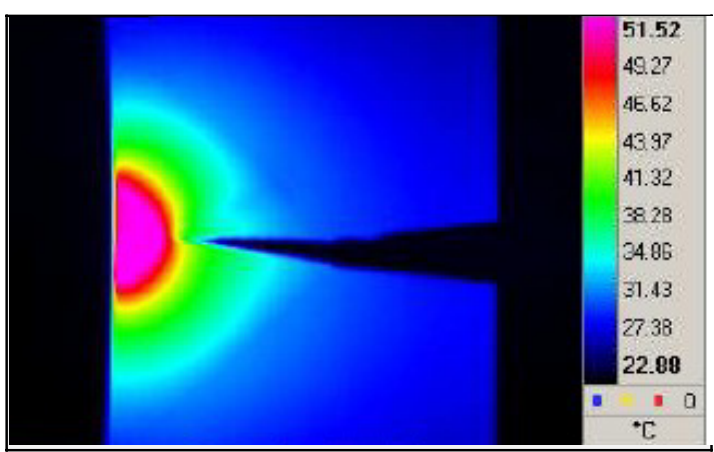

b)

Fig. 4. Temperature field evolution during fracture test: at the crack propagation beginning a), during the propagation b) and at fracture c).

On figure 4, a map of temperature fields is given for three moments throughout the fracture test: at the beginning, about the middle and at the end of the crack propagation. Isotherms appear like a boomerang-shaped with an orientation of approximately $50^{\circ} \mathrm{C}$ relatively to the crack direction. At the moment, we do not have 
enough information at our disposal to relate this thermal gradients shape to the predicted analytical transformation phase.

Figure 5 presents crack tip temperature evolution. Firstly, this last one is constant, then increases a little to reach a plateau. This plateau corresponds to propagation initiation. Then, temperature at crack tip start rising as crack opening, and reaches a maximum $\left(68^{\circ} \mathrm{C}\right)$ then falls down abruptly (about of $40^{\circ} \mathrm{C}$ ) at fracture. Transformation temperatures are unknown but we assume that the martensitic transformation begins just after the plateau, at the temperature increase.

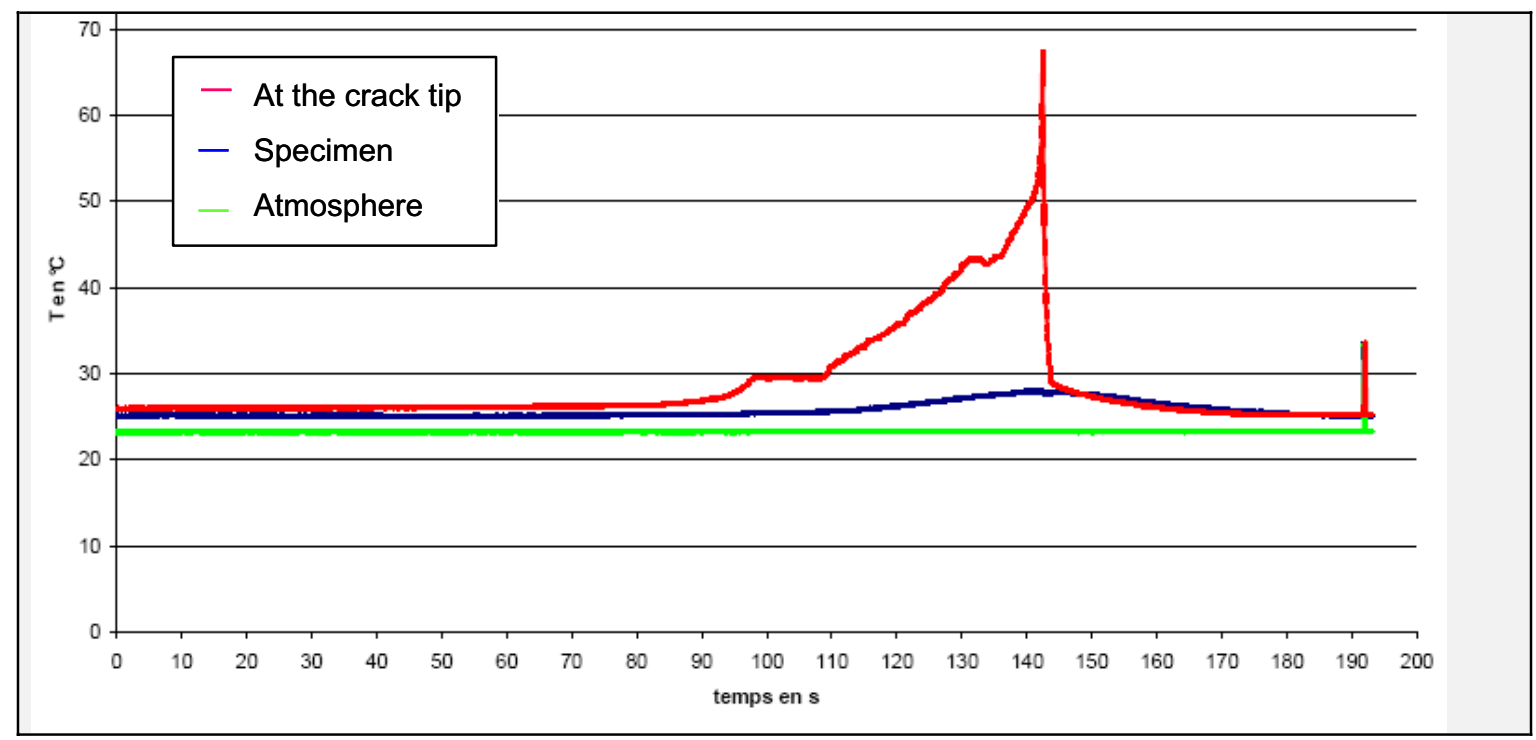

Fig. 5. Crack tip temperature evolution versus time.

\subsection{Future measures of displacement field with images correlation}

In addition to temperature field measurements, coupled displacement field measurements will be implemented by Digital Image Correlation (DIC). This in-situ optical method will allow building strain field map by tracking and correlating a random pattern on the specimen surface. It will inform on phase transformation regions thanks to strain localization observations and will permits to compare its with predicted transformation regions. Besides, the final aim is to attempt a correlation between thermal fields and kinematic fields.

\section{Conclusions}

This work constitutes an introduction into the world of fracture mechanics for phase transformation material such as SMAs. The purpose is to better understand fracture mechanisms for these alloys and attempt to supply a predictive tool. Experimental parts need to be developed in the next months.

\section{References}

[1] Freed Y., Banks-Sill L., J. Mech. Phys. Solids 55, 2157, (2007)

[2] Robertson S.W., Ritchie R.O., Biomaterial 28, 700, (2007)

[3] Panoskaltis V.P., Bahuguna S., Soldatos S., Int. J. Non-Linear Mech. 39, 709, (2004)

[4] Raniecki B., Lexcellent C., Eur. J. Mech. A/Solids 17, 185, (1998)

[5] Bouvet C., Calloch S., Lexcellent C., Eur. J. Mech. Solids 61, 23, (2004)

[6] Bouvet C., Calloch S., Lexcellent C., Eur. J. Eng. Mater. Technol. 124, 665, (2002)

[7] Laydi M.R., Lexcellent C., Math. Mech. Solids (2008)

[8] Lexcellent C., Thiebaud F., Scripta Mat. 59, 321, (2008) 